\title{
Electroweak and QCD corrections to top-pair hadroproduction in association with heavy bosons
}

\author{
S. Frixione, ${ }^{a}$ V. Hirschi, ${ }^{b}$ D. Pagani, ${ }^{c}$ H.-S. Shao ${ }^{a}$ and M. Zaro ${ }^{d, e}$ \\ ${ }^{a}$ PH Department, TH Unit, CERN, \\ CH-1211 Geneva 23, Switzerland \\ ${ }^{b}$ SLAC, National Accelerator Laboratory, \\ 2575 Sand Hill Road, Menlo Park, CA 94025-7090, U.S.A. \\ ${ }^{c}$ Centre for Cosmology, Particle Physics and Phenomenology (CP3), \\ Université Catholique de Louvain, \\ B-1348 Louvain-la-Neuve, Belgium \\ ${ }^{d}$ Sorbonne Universités, UPMC Université Paris 06, UMR 7589, LPTHE, \\ F-75005, Paris, France \\ ${ }^{e}$ CNRS, UMR 7589, LPTHE, \\ F-75005, Paris, France \\ E-mail: stefano.frixione@cern.ch, vahirsch@slac.stanford.edu, \\ davide.pagani@uclouvain.be, huasheng.shao@cern.ch, \\ marco.zaro@lpthe.jussieu.fr
}

Abstract: We compute the contribution of order $\alpha_{S}^{2} \alpha^{2}$ to the cross section of a topantitop pair in association with at least one heavy Standard Model boson $-Z, W^{ \pm}$, and Higgs - by including all effects of QCD, QED, and weak origin and by working in the automated MADGRAPH5_AMC@NLO framework. This next-to-leading order contribution is then combined with that of order $\alpha_{S}^{3} \alpha$, and with the two dominant lowest-order ones, $\alpha_{S}^{2} \alpha$ and $\alpha_{S} \alpha^{2}$, to obtain phenomenological results relevant to a 8,13 , and $100 \mathrm{TeV} p p$ collider.

KeYwords: NLO Computations, Hadronic Colliders

ARXIV EPRINT: 1504.03446 


\section{Contents}

1 Introduction 1

2 Definitions and calculation details 2

3 Results 4

3.1 Inclusive rates 6

$\begin{array}{lll}3.2 & \text { Differential distributions } & 12\end{array}$

4 Conclusions and outlook $\quad 14$

\section{Introduction}

The hadroproduction cross sections for a top pair in association with a Standard Model (SM) heavy boson $V$ are interesting in many respects, and are thus actively studied by theorists and experimenters alike. In the context of the SM, they constitute excellent tests for the EW theory and for its perturbative predictions, and a unique and direct probe of the $t \bar{t} V$ couplings $\lambda_{t \bar{t} V}$ in the cases $V=Z$ and $V=H$. On the other hand, by assuming the correctness of the SM description of $t \bar{t} W^{ \pm}$and $t \bar{t} Z$ production, these processes feature prominently as backgrounds in several searches for beyond-the-SM (BSM) signals, typically characterised by multi-lepton signatures (e.g. same-sign di-leptons or tri-leptons final states), including SUSY [1-3], extra dimensions [4], and models with heavy top-quark partners [5]. Needless to say, they are also backgrounds to $t \bar{t} H$ production itself, again in the case of multi-lepton signatures.

Experimentally, evidence of $t \bar{t} V$ production is hard to obtain even at the very high LHC c.m. energies, owing to the smallness of the rates and/or to background contaminations. Measurements of the $t \bar{t} W^{ \pm}$and $t \bar{t} Z$ cross sections extracted from Run-I data have been reported by both ATLAS and CMS [6-10]; they are affected by very large uncertainties, which are statistically dominated (although not overwhelmingly so). Conversely, the $t \bar{t} H$ cross section has not yet been measured at the LHC; however, exclusion limits on the SM value have been established with several searches that employ a variety of Higgs decay channels [11-18].

From the theoretical point of view, the calculation of $t \bar{t} V$ cross sections used to represent a very challenging problem beyond the leading order (LO); such higher-order calculations have a strong phenomenological motivation, since these processes are characterised by large $K$ factors. These computational problems have been fully solved at the next-toleading order (NLO) by the advent of modern automation techniques; nowadays, results accurate to the NLO in QCD are straightforwardly available (see e.g. refs. [19-23] for $t \bar{t} Z$ production, refs. [20, 23, 24] for $t \bar{t} W^{ \pm}$production, and refs. [25-30] for $t \bar{t} H$ production); the most recent among them include matching to parton showers. 
While at present the precision of the theoretical predictions is thus sufficient for all kind of phenomenological applications, since one is dominated by experimental uncertainties, the Run II of the LHC, with both energy and luminosity larger than in Run I, might soon change the situation, whence the need to increase the precision of the perturbative predictions. At fixed order, this can be done in two ways: by computing either the NNLO QCD corrections, or the NLO electroweak (EW) ones; these, as a rule of thumb, are believed to have comparable numerical impacts. Calculations at the NNLO in QCD for processes of the complexity of $t \bar{t} V$ production are beyond the scope of the currently available technology. This is not the case for NLO EW corrections, and the aim of this paper is to compute them. We point out that, for what concerns $t \bar{t} H$ production, purely-weak and EW corrections have been presented in refs. [31,32] respectively, while they are currently unknown for $t \bar{t} Z$ and $t \bar{t} W^{ \pm}$production, and are considered in this paper for the first time.

We remind the reader that, in the context of a perturbative expansion where both $\alpha_{S}$ and $\alpha$ are treated as small parameters (called a mixed-coupling scenario in ref. [33]), what is meant by "NLO EW corrections" is conventional, and might lead to ambiguities. A more precise terminology, advocated in ref. [31], uses "leading NLO" and "second-leading NLO" contributions to denote what are traditionally called NLO QCD and NLO EW corrections, ${ }^{1}$ respectively. In the case of $t \bar{t} V$ production, these correspond to the $\mathcal{O}\left(\alpha_{S}^{3} \alpha\right)$ and $\mathcal{O}\left(\alpha_{S}^{2} \alpha^{2}\right)$ contributions to the cross sections. In the phenomenological results to be presented below, we shall include the leading and second-leading LO terms as well, of $\mathcal{O}\left(\alpha_{S}^{2} \alpha\right)$ and $\mathcal{O}\left(\alpha_{S} \alpha^{2}\right)$ respectively.

Our work is performed within the MadGraph5_AMC@NLO framework [33]. Apart from the novelty of the physics results presented here, this paper constitutes the first application of such a code to the fully-automated computation of cross sections that require the subtraction of QED singularities. We stress, in particular, that no aspect of the code has been designed or optimised in order to deal specifically with $t \bar{t} V$ production, in keeping with the general strategy that underpins MADGRAPH5_AMC@NLO. We also remark that the calculation of EW corrections and their automation is receiving a growing attention from the high-energy physics community (see e.g. refs. [34-37] for recent results).

This paper is organised as follows. In section 2 we introduce our notation, and briefly describe the calculation as is performed by MADGRAPH5_AMC@NLO. In section 3 we present results for total cross sections and sample differential distributions. We summarise our findings in section 4.

\section{Definitions and calculation details}

We adopt, as far as it is possible, the definitions and notations introduced in ref. [31]. A generic observable $\Sigma\left(\alpha_{S}, \alpha\right)$ in $t \bar{t} V$ production can be written at the $\mathrm{LO}$ as follows:

$$
\begin{aligned}
\Sigma_{t \bar{t} V}^{(\mathrm{LO})}\left(\alpha_{S}, \alpha\right) & =\alpha_{S}^{2} \alpha \Sigma_{3,0}+\alpha_{S} \alpha^{2} \Sigma_{3,1}+\alpha^{3} \Sigma_{3,2} \\
& \equiv \Sigma_{\mathrm{LO}, 1}+\Sigma_{\mathrm{LO}, 2}+\Sigma_{\mathrm{LO}, 3} .
\end{aligned}
$$

\footnotetext{
${ }^{1}$ The standard definition of the latter neglects the contributions due to the radiation of an extra heavy boson. See section 2 and ref. [31] for more details.
} 
This equation implicitly defines the leading, second-leading, and third-leading $\Sigma_{\mathrm{LO}, q+1}$ contributions in terms of the coefficients $\Sigma_{3, q}$ that appear naturally in a mixed-coupling expansion (see section 2.4 of ref. [33] for more details). Analogously, at the NLO one has:

$$
\begin{aligned}
\Sigma_{t \bar{t} V}^{(\mathrm{NLO})}\left(\alpha_{S}, \alpha\right) & =\alpha_{S}^{3} \alpha \Sigma_{4,0}+\alpha_{S}^{2} \alpha^{2} \Sigma_{4,1}+\alpha_{S} \alpha^{3} \Sigma_{4,2}+\alpha^{4} \Sigma_{4,3} \\
& \equiv \Sigma_{\mathrm{NLO}, 1}+\Sigma_{\mathrm{NLO}, 2}+\Sigma_{\mathrm{NLO}, 3}+\Sigma_{\mathrm{NLO}, 4} .
\end{aligned}
$$

We shall retain in our computation the two dominant terms at the LO and NLO, namely $\Sigma_{\mathrm{LO}, 1}, \Sigma_{\mathrm{LO}, 2}, \Sigma_{\mathrm{NLO}, 1}$, and $\Sigma_{\mathrm{NLO}, 2}$.

The LO contribution $\Sigma_{\mathrm{LO}, 2}$ vanishes in the case of $t \bar{t} W^{ \pm}$production, and is numerically rather small in the cases of the cross sections for electrically-neutral bosons, $t \bar{t} Z$ and $t \bar{t} H$. As discussed in ref. [31], the latter two final states stem at this perturbative order from partonic processes with a $b \bar{b}$ initial state, ${ }^{2}$ if only weak effects are considered. However, when one includes QED effects, diagrams with an initial-state photon contribute as well. Partonic processes with one incoming photon also contribute to the second-leading NLO term $\Sigma_{\mathrm{NLO}, 2}$. While this fact does not pose any problems at the level of short-distance computations, it requires one to use PDFs that feature photon densities, and that incorporate QED evolution. From this viewpoint, the situation is less than satisfactory. The only modern such PDF set is NNPDF2.3QED [38], which has the disadvantage of treating QED effects only at the leading order. While formally this degrades the NLO accuracy of (part of) our computation, in practice it does not constitute a major problem, given that the photon density is anyhow rather poorly determined at present. In the following, we shall be assessing carefully the impact of PDF uncertainties on our predictions, both by employing the NNPDF prescription, and by artificially setting the photon density equal to zero. We point out that another consequence of having QED-LL-evolved PDFs is the possibility of using an arbitrary scheme for the finite parts of the initial-state QED subtractions. In this paper, we have adopted the $\overline{\mathrm{MS}}$ scheme, and have refrained from studying the dependence of our results on the QED scheme choice for the PDFs.

As was already mentioned, all of our results are obtained by means of the automatic code MADGRAPH5_AMC@NLO, which contains all ingredients relevant to the computations of LO and NLO cross sections, with or without matching to parton showers. NLO results not matched to parton showers are obtained by adopting the FKS method [39, 40] for the subtraction of the singularities of the real-emission matrix elements (automated in the module MADFKS [41]), and the OPP [42] or Tensor Integral Reduction (TIR [43, 44]) procedures for the computation of the one-loop matrix elements (automated in the module MadLoop [20,33], which makes use of CutTools [45] with OPP and of IREGI [46] with TIR, and of an in-house implementation of the representation proposed in ref. [47] (OPENLoops)). The automation of mixed-coupling expansions has now been fully achieved also in MADFKS, at variance with the situation of ref. [31], and the present paper is part of the ongoing validation effort. We have performed all of the self-consistency checks available in MADGRAPH5_AMC@NLO, which are discussed in ref. [33] (see in particular section 2.4.2

\footnotetext{
${ }^{2}$ Under the assumption that the CKM matrix be diagonal, as is done here. Light-quark initial states $q \bar{q}$ are possible when this assumption is relaxed, but then the corresponding contributions are CKM-suppressed.
} 
of that paper for what concerns one-loop matrix elements). Here, we mention explicitly the independence of the cross section of the values taken by the FKS subtraction parameters [39] $\xi_{\text {cut }}$ and $\delta_{I}$, which is directly relevant to the newly-implemented QED subtractions. Furthermore, we have computed to high numerical accuracy $(\mathcal{O}(0.1 \%))$ the LO and NLO contributions both separately and in the course of the same numerical simulation, and found full agreement between these two procedures. We also remark that the secondleading NLO term $\Sigma_{\mathrm{NLO}, 2}$ can be organised internally by MADGRAPH5_AMC@NLO in two different ways, which correspond to seeing it (in an unphysical manner [31]) as either an EW correction to $\Sigma_{\mathrm{LO}, 1}$ or a $\mathrm{QCD}$ correction to $\Sigma_{\mathrm{LO}, 2}$; we have verified that these two ways lead to the same numerical results.

The notation of eqs. (2.1) and (2.2) may be unfamiliar to most readers. Given that in this paper we restrict ourselves to the computation of the two dominant terms at each perturbative order, one can introduce an alternative notation, which is less precise (see ref. [31] for more details) but rather consistent with what has been used in the literature so far. Such an alternative labeling scheme, which we shall adopt extensively in section 3, is summarised in table 1. We stress that the two lines at the bottom of that table imply:

$$
\Sigma_{\mathrm{NLO}, 2}=\Sigma_{\mathrm{NLO} \mathrm{EW}}+\Sigma_{\mathrm{HBR}}
$$

with:

$$
\begin{aligned}
\Sigma_{\mathrm{NLO} \text { EW }}(t \bar{t} V) & =\alpha_{S}^{2} \alpha^{2} \sum_{X \neq W^{ \pm}, Z, H} \Sigma_{4,1}(t \bar{t} V+X), \\
\Sigma_{\mathrm{HBR}}(t \bar{t} V) & =\alpha_{S}^{2} \alpha^{2} \sum_{X=W^{ \pm}, Z, H} \Sigma_{4,1}(t \bar{t} V+X) .
\end{aligned}
$$

The two terms on the r.h.s. of eq. (2.3) are both finite and theoretically well defined, and we shall present the corresponding results separately (rather than only for their sum $\left.\Sigma_{\mathrm{NLO}, 2}\right)$. In the vast majority of the results available in the literature, the analogue of the HBR contribution is simply ignored, on the basis of the fact that its final states are distinguishable from those relevant to eq. (2.4). Unfortunately, such an argument is rather unphysical, because it cannot be quantified unless a proper study is made that uses the decay products of the vector bosons, and suitable acceptance cuts are imposed on their momenta. A fully realistic simulation of this kind would be particularly important were the experimental results quoted for cross sections exclusive in exactly one heavy boson. Note, finally, that a very similar argument could be made in the case of the radiation of a photon (of sufficient hardness), whereas typically cross sections that include QED corrections are computed fully inclusively in any extra photon (as we do in eq. (2.4)).

\section{Results}

In this section we present our predictions for inclusive rates relevant to the production of $t \bar{t} H, t \bar{t} Z, t \bar{t} W^{+}$, and $t \bar{t} W^{-}$at a $p p$ collider with a c.m. energy of $8 \mathrm{TeV}$ (LHC Run I), $13 \mathrm{TeV}$ (LHC Run II), and $100 \mathrm{TeV}$. In the case of the LHC Run II, we shall also study 


\begin{tabular}{|l|l|}
\hline Label & Meaning \\
\hline LO QCD & LO, 1 \\
NLO QCD & NLO, 1 \\
LO EW & LO, 2 \\
NLO EW & NLO, 2 ; no $p p \rightarrow t \bar{t} V_{1} V_{2}$ \\
HBR & NLO, 2 ; only $p p \rightarrow t \bar{t} V_{1} V_{2}$ \\
\hline
\end{tabular}

Table 1. Shorthand notation used in section 3. $V_{1}$ and $V_{2}$ stand for a Higgs, a $W^{ \pm}$, or a $Z$ boson. HBR is an acronym for Heavy Boson Radiation, and for a given $V_{1}$ understands the sum over $V_{2}$. The reader is encouraged to check section 2 for the precise definitions of all the quantities involved.

the four production processes at the level of several differential distributions. Furthermore, for such a c.m. energy we shall consider the implications of a "boosted" regime, effectively obtained by imposing the following final-state cuts:

$$
p_{T}(t) \geq 200 \mathrm{GeV}, \quad p_{T}(\bar{t}) \geq 200 \mathrm{GeV}, \quad p_{T}(V) \geq 200 \mathrm{GeV} .
$$

In HBR processes, the transverse momentum of the vector boson denoted by $X$ in eq. (2.5) is not constrained; this implies that, in the case of identical particles $(X=V)$, a single vector boson fulfilling the last condition in eq. (3.1) is sufficient for the corresponding event to contribute to the cross section. While a high- $p_{T}$ regime might be advocated in the context of Higgs searches [48-50] to increase the relative strength of the signal, in the present case it is interesting regardless of the nature of the associated heavy boson, because it is known to enhance the impact of EW effects through large Sudakov logarithms [51-54]. Thus, it allows one to gauge directly the impact of EW corrections where they should matter most, and hence to assess the reliability of predictions that include only NLO QCD effects.

We have chosen the particle masses as follows:

$$
\begin{aligned}
m_{t} & =173.3 \mathrm{GeV}, & m_{H} & =125 \mathrm{GeV}, \\
m_{W} & =80.385 \mathrm{GeV}, & m_{Z} & =91.188 \mathrm{GeV} .
\end{aligned}
$$

All widths are set equal to zero, and the massive modes and Yukawas are renormalised onshell. We point out that these settings are not hard-coded in MADGRAPH5_AMC@NLO, but are inherited [33] from the adopted UFO [55] model. We have chosen the NNPDF2.3QED PDF set [38] (particularly for the reasons discussed in section 2) that is associated with the value

$$
\alpha_{S}\left(m_{Z}\right)=0.118 .
$$

Our default EW scheme is the $\alpha\left(m_{Z}\right)$ scheme, where we set:

$$
\frac{1}{\alpha\left(m_{Z}\right)}=128.93 \text {. }
$$

We shall also present results in the $G_{\mu}$ scheme, where:

$$
G_{\mu}=1.16639 \cdot 10^{-5} \quad \longrightarrow \quad \frac{1}{\alpha}=132.23 .
$$


The central values of the renormalisation $\left(\mu_{R}\right)$ and factorisation $\left(\mu_{F}\right)$ scales have been taken equal to the reference scale:

$$
\mu=\frac{H_{T}}{2} \equiv \frac{1}{2} \sum_{i} \sqrt{m_{i}^{2}+p_{T}^{2}(i)},
$$

where the sum runs over all final-state particles. The theoretical uncertainties due to the $\mu_{R}$ and $\mu_{F}$ dependencies have been evaluated by varying these scales independently in the range:

$$
\frac{1}{2} \mu \leq \mu_{R}, \mu_{F} \leq 2 \mu,
$$

and by taking the envelope of the resulting predictions; the value of $\alpha$ is kept fixed. In this work, we have limited ourselves to considering the scale dependence of $\Sigma_{\mathrm{LO}, 1}$ and $\Sigma_{\mathrm{NLO}, 1}$, which corresponds to what is usually identified with the scale uncertainty of the QCD cross section. We point out that the calculation of this theory systematics does not entail any independent runs, being performed through the exact reweighting technique introduced in ref. [56], which is fully automated in MADGRAPH5_AMC@NLO. The PDF uncertainties are computed, again through reweighting, by following the NNPDF methodology [57]; we report the $68 \%$ CL symmetric interval (that is the one that contains only 68 replicas out of a total of a hundred; this is done in order to avoid the problem of outliers, which is severe in this case owing to the photon PDF [38])

We stress that, because of the choice of PDFs made in this paper, the present results for $t \bar{t} H$ production would not be exactly identical to those of ref. [31] even if QED effects were ignored. However, the differences are tiny, so that a direct comparison between the $t \bar{t} H$ results of this paper and those of ref. [31] is possible, which allows one to assess the impact of QED-only corrections.

\subsection{Inclusive rates}

We begin by reporting, in table 2 , the results relevant to the individual contributions that enter the definition of a given HBR cross section. As is implied by eq. (2.5), by summing the relevant entries of table 2 one obtains the desired HBR rate. For example, in the case of $t \bar{t} H$ production:

$$
\sigma_{\mathrm{HBR}}(t \bar{t} H)=\sigma(t \bar{t} H H)+\sigma(t \bar{t} H Z)+\sigma\left(t \bar{t} H W^{+}\right)+\sigma\left(t \bar{t} H W^{-}\right),
$$

and analogously for the other processes. Note that HBR cross sections are inclusive by definition, and cannot be summed; this is evident if one considers that one given contribution may enter in more than one HBR rate (e.g. $\sigma(t \bar{t} H Z)$ contributes to the HBR's of both $t \bar{t} H$ and $t \bar{t} Z$ ). The entries of table 2 have a relative integration error of about $0.1 \%$.

We now present, in turn, the results for the total rates relevant to $t \bar{t} H, t \bar{t} Z, t \bar{t} W^{+}$, and $t \bar{t} W^{-}$production. Each of these processes corresponds to a set of two tables: tables 3 and 4 for $t \bar{t} H$, tables 5 and 6 for $t \bar{t} Z$, tables 7 and 8 for $t \bar{t} W^{+}$, and tables 9 and 10 for $t \bar{t} W^{-}$. In the first table of each set we give the values, in $\mathrm{pb}$, of the various contributions to the total cross section, namely LO QCD, NLO QCD, LO EW, NLO EW, and HBR; at a given c.m. energy, these results have an integration error which is at most $0.1 \%$ times the 


\begin{tabular}{|l|ccc|}
\hline$\sigma(\mathrm{pb})$ & $8 \mathrm{TeV}$ & $13 \mathrm{TeV}$ & $100 \mathrm{TeV}$ \\
\hline$t \bar{t} H H$ & $1.640 \cdot 10^{-4}$ & $6.947 \cdot 10^{-4}$ & $6.078 \cdot 10^{-2}$ \\
$t \bar{t} H Z$ & $2.831 \cdot 10^{-4}$ & $1.214 \cdot 10^{-3}$ & $1.212 \cdot 10^{-1}$ \\
$t \bar{t} H W^{+}$ & $2.918 \cdot 10^{-4}$ & $8.996 \cdot 10^{-4}$ & $1.982 \cdot 10^{-2}$ \\
$t \bar{t} H W^{-}$ & $1.139 \cdot 10^{-4}$ & $4.074 \cdot 10^{-4}$ & $1.366 \cdot 10^{-2}$ \\
$t \bar{t} Z Z$ & $3.373 \cdot 10^{-4}$ & $1.385 \cdot 10^{-3}$ & $1.209 \cdot 10^{-1}$ \\
$t \bar{t} Z W^{+}$ & $5.036 \cdot 10^{-4}$ & $1.711 \cdot 10^{-3}$ & $4.634 \cdot 10^{-2}$ \\
$t \bar{t} Z W^{-}$ & $1.919 \cdot 10^{-4}$ & $7.455 \cdot 10^{-4}$ & $3.084 \cdot 10^{-2}$ \\
$t \bar{t} W^{+} W^{-}$ & $1.618 \cdot 10^{-3}$ & $7.066 \cdot 10^{-3}$ & $7.747 \cdot 10^{-1}$ \\
\hline
\end{tabular}

Table 2. Total rates for the individual contributions to HBR cross sections.

LO QCD cross section ${ }^{3}$ relevant to that energy. The two contributions labelled with "EW" are also computed by setting the photon density equal to zero, as explained in section 2 . In the case of the $13 \mathrm{TeV}$ LHC, we also give (in parentheses) the rates within the cuts of eq. (3.1). The second table of each set displays the value of the ratios:

$$
\delta_{\mathrm{X}}=\frac{\sigma_{\mathrm{X}}}{\sigma_{\mathrm{LO} \mathrm{QCD}}}
$$

with X equal to NLO QCD, LO EW, NLO EW, and HBR. In other words, for any given column the entry in the $n^{\text {th }}$ row of the second table is equal to the ratio of the entry in the $(n+1)^{t h}$ row of the first table over the entry in the first row of that table. Except for HBR, the results for the ratios $\delta$ are associated with uncertainties. These fractional uncertainties are computed by using eq. (3.10), with the numerator set equal to the maximum and minimum of either the scale or the PDF envelope, and the denominator always computed with central scales and PDFs. Note that the denominator is a LO quantity, at variance with what is done usually in QCD where the central NLO cross section is used; the present choice allows one to treat $\mathrm{QCD}$ and $\mathrm{EW}$ effects on a more equal footing in the context of a mixed-coupling expansion. In the case of NLO QCD, the uncertainties quoted in the tables are due to scale variations (leftmost errors) and PDF variations (rightmost errors); in the case of the LO and NLO EW contributions, to PDF variations.

The results for the total cross sections exhibit a few features common to all four processes considered here. Firstly, the leading NLO term (NLO QCD) is very large, and grows with the collider energy. Its impact is particularly striking in the case of $t \bar{t} W^{ \pm}$production, owing to the opening at the NLO of partonic channels $(q g)$ that feature a gluon PDF, while no initial-state gluon is present at the LO - in the case of $t \bar{t} H$ and $t \bar{t} Z$ production, one has $g g$-initiated partonic processes already at the Born level. As a consequence of this, the scale uncertainty, which is relatively large for all processes, becomes extremely significant in $t \bar{t} W^{ \pm}$production of increasing hardness (large c.m. energy or boosted regime), where it is predominantly of LO-type because of the growing contributions of $q g$-initiated partonic processes. In all cases, the PDF uncertainties of the NLO QCD term are smaller than those

\footnotetext{
${ }^{3}$ The typical errors are such that the statistical uncertainties affect the last digit of the results quoted in the tables.
} 


\begin{tabular}{|c|ccc|}
\hline$t \bar{t} H: \sigma(\mathrm{pb})$ & $8 \mathrm{TeV}$ & $13 \mathrm{TeV}$ & $100 \mathrm{TeV}$ \\
\hline LO QCD & $9.685 \cdot 10^{-2}$ & $3.617 \cdot 10^{-1}\left(1.338 \cdot 10^{-2}\right)$ & 23.57 \\
NLO QCD & $2.507 \cdot 10^{-2}$ & $1.073 \cdot 10^{-1}\left(3.230 \cdot 10^{-3}\right)$ & 9.61 \\
\hline LO EW & $1.719 \cdot 10^{-3}$ & $4.437 \cdot 10^{-3}\left(3.758 \cdot 10^{-4}\right)$ & $1.123 \cdot 10^{-2}$ \\
LO EW no $\gamma$ & $-2.652 \cdot 10^{-4}$ & $-1.390 \cdot 10^{-3}\left(-2.452 \cdot 10^{-5}\right)$ & $-1.356 \cdot 10^{-1}$ \\
\hline NLO EW & $-5.367 \cdot 10^{-4}$ & $-4.408 \cdot 10^{-3}\left(-1.097 \cdot 10^{-3}\right)$ & $-6.261 \cdot 10^{-1}$ \\
NLO EW no $\gamma$ & $-7.039 \cdot 10^{-4}$ & $-4.919 \cdot 10^{-3}\left(-1.131 \cdot 10^{-3}\right)$ & $-6.367 \cdot 10^{-1}$ \\
\hline HBR & $8.529 \cdot 10^{-4}$ & $3.216 \cdot 10^{-3}\left(2.496 \cdot 10^{-4}\right)$ & $2.154 \cdot 10^{-1}$ \\
\hline
\end{tabular}

Table 3. Contributions, as defined in table 1, to the total rate (in pb) of $t \bar{t} H$ production, for three different collider energies. The results in parentheses are relevant to the boosted scenario, eq. (3.1).

\begin{tabular}{|c|ccc|}
\hline$t \bar{t} H: \delta(\%)$ & $8 \mathrm{TeV}$ & $13 \mathrm{TeV}$ & $100 \mathrm{TeV}$ \\
\hline NLO QCD & $25.9_{-11.1}^{+5.4} \pm 3.5$ & $29.7_{-11.1}^{+6.8} \pm 2.8\left(24.2_{-10.6}^{+4.8} \pm 4.5\right)$ & $40.8_{-9.1}^{+9.3} \pm 1.0$ \\
\hline LO EW & $1.8 \pm 1.3$ & $1.2 \pm 0.9(2.8 \pm 2.0)$ & $0.0 \pm 0.2$ \\
LO EW no $\gamma$ & $-0.3 \pm 0.0$ & $-0.4 \pm 0.0(-0.2 \pm 0.0)$ & $-0.6 \pm 0.0$ \\
\hline NLO EW & $-0.6 \pm 0.1$ & $-1.2 \pm 0.1(-8.2 \pm 0.3)$ & $-2.7 \pm 0.0$ \\
NLO EW no $\gamma$ & $-0.7 \pm 0.0$ & $-1.4 \pm 0.0(-8.5 \pm 0.2)$ & $-2.7 \pm 0.0$ \\
\hline HBR & 0.88 & $0.89(1.87)$ & 0.91 \\
\hline
\end{tabular}

Table 4. Same as in table 3, but given as fractions of corresponding LO QCD cross sections. Scale (for NLO QCD) and PDF uncertainties are also shown.

due to the hard scales, and decrease with the c.m. energy. Secondly, the contributions due to processes with initial-state photons are quite large at the LO (except for $t \bar{t} W^{ \pm}$production, which has a LO EW cross section identically equal to zero), but constitute only a small fraction of the total at the NLO. This is due to the fact that LO EW processes proceed only through two types of initial states, namely $\gamma g$ and $b \bar{b}$, whereas NLO EW ones have richer incoming-parton luminosities. Thirdly, as a consequence of the previous point, the uncertainty of the photon density only marginally increases (if at all) the total PDF uncertainty that affects the NLO EW term, while it constitutes a dominant factor at the LO EW level (for $t \bar{t} H$ and $t \bar{t} Z$ ).

Other aspects characterise differently the four $t \bar{t} V$ processes. The relative importance of NLO EW contributions w.r.t. the NLO QCD ones increases with energy in the cases of $t \bar{t} H$ and $t \bar{t} Z$ production, while it decreases for $t \bar{t} W^{ \pm}$production. At the $8-\mathrm{TeV}$ LHC, NLO EW terms have the largest impact on $t \bar{t} W^{+}$(about $17 \%$ of the NLO QCD ones), and the smallest on $t \bar{t} H(2.7 \%)$. This is reflected in the fact that for $t \bar{t} W^{ \pm}$production the NLO EW effects are barely within the NLO QCD scale uncertainty band; conversely, for $t \bar{t} H$ and $t \bar{t} Z$ production NLO EW contributions are amply within the NLO QCD uncertainties. By imposing at the NLO EW level and at the 13-TeV LHC the boosted conditions enforced by eq. (3.1), the change w.r.t. the non-boosted scenario is largest in the case of $t \bar{t} H$ production 


\begin{tabular}{|c|ccc|}
\hline$t \bar{t} Z: \sigma(\mathrm{pb})$ & $8 \mathrm{TeV}$ & $13 \mathrm{TeV}$ & $100 \mathrm{TeV}$ \\
\hline LO QCD & $1.379 \cdot 10^{-1}$ & $5.282 \cdot 10^{-1}\left(1.955 \cdot 10^{-2}\right)$ & 37.69 \\
NLO QCD & $5.956 \cdot 10^{-2}$ & $2.426 \cdot 10^{-1}\left(7.856 \cdot 10^{-3}\right)$ & 18.99 \\
\hline LO EW & $6.552 \cdot 10^{-4}$ & $-2.172 \cdot 10^{-4}\left(4.039 \cdot 10^{-4}\right)$ & $-4.278 \cdot 10^{-1}$ \\
LO EW no $\gamma$ & $-1.105 \cdot 10^{-3}$ & $-5.771 \cdot 10^{-3}\left(-6.179 \cdot 10^{-5}\right)$ & $-5.931 \cdot 10^{-1}$ \\
\hline NLO EW & $-4.540 \cdot 10^{-3}$ & $-2.017 \cdot 10^{-2}\left(-2.172 \cdot 10^{-3}\right)$ & -1.974 \\
NLO EW no $\gamma$ & $-5.069 \cdot 10^{-3}$ & $-2.158 \cdot 10^{-2}\left(-2.252 \cdot 10^{-3}\right)$ & -2.036 \\
\hline HBR & $1.316 \cdot 10^{-3}$ & $5.056 \cdot 10^{-3}\left(4.162 \cdot 10^{-4}\right)$ & $3.192 \cdot 10^{-1}$ \\
\hline
\end{tabular}

Table 5. Same as in table 3, for $t \bar{t} Z$ production.

\begin{tabular}{|c|ccc|}
\hline$t \bar{t} Z: \delta(\%)$ & $8 \mathrm{TeV}$ & $13 \mathrm{TeV}$ & $100 \mathrm{TeV}$ \\
\hline NLO QCD & $43.2_{-15.9}^{+12.8} \pm 3.6$ & $45.9_{-15.5}^{+13.2} \pm 2.9\left(40.2_{-15.0}^{+11.1} \pm 4.7\right)$ & $50.4_{-10.9}^{+11.4} \pm 1.1$ \\
\hline LO EW & $0.5 \pm 0.9$ & $0.0 \pm 0.7(2.1 \pm 1.6)$ & $-1.1 \pm 0.2$ \\
LO EW no $\gamma$ & $-0.8 \pm 0.1$ & $-1.1 \pm 0.0(-0.3 \pm 0.0)$ & $-1.6 \pm 0.0$ \\
\hline NLO EW & $-3.3 \pm 0.3$ & $-3.8 \pm 0.2(-11.1 \pm 0.5)$ & $-5.2 \pm 0.1$ \\
NLO EW no $\gamma$ & $-3.7 \pm 0.1$ & $-4.1 \pm 0.1(-11.5 \pm 0.3)$ & $-5.4 \pm 0.0$ \\
\hline HBR & 0.95 & $0.96(2.13)$ & 0.85 \\
\hline
\end{tabular}

Table 6. Same as in table 4 , for $t \bar{t} Z$ production.

(by a factor equal to about 6.8 ); $t \bar{t} Z$ and $t \bar{t} W^{ \pm}$behave similarly, with enhancement factors in the range 2.5-3. However, for all processes the boosted kinematics are such that the NLO EW terms are equal or larger than the scale uncertainties that affect the corresponding NLO QCD terms. For both of the processes which have a non-trivial LO EW cross section $(t \bar{t} H$ and $t \bar{t} Z)$, the $b \bar{b}$ - and $\gamma g$-initiated contributions tend to cancel each other. In the case of $t \bar{t} H$, an almost complete (and accidental) cancellation (relative to the LO QCD term) occurs at a c.m. energy of $100 \mathrm{TeV}$, while for $t \bar{t} Z$ it so does at the much lower LHC Run II energy. This implies that the impact of EW effects at the $13-\mathrm{TeV}$ LHC is more important in the case of $t \bar{t} Z$ than for $t \bar{t} H$ production, given that for the latter process the LO and NLO contributions tend to cancel in the sum at this collider energy. However, it is necessary to keep in mind the observation about the uncertainties induced on the LO EW cross section by the photon density: a better determination of such a PDF would be desirable, in order to render the statement above quantitatively more precise. Finally for $t \bar{t} H$ production, by comparing the results of table 4 relevant to the NLO EW terms with those of table 6 of ref. [31] relevant to the weak-only contributions to the NLO cross section, one sees that the relative impact of QED effects decreases with the c.m. energy and is rather negligible in the boosted scenario, as expected. These QED effects have the opposite sign w.r.t. those of weak origin, and can be as large as half of the latter at the LHC Run I.

As far as the HBR cross sections are concerned, some general considerations about the various mechanisms that govern the (partial) compensation between these terms and 


\begin{tabular}{|c|ccc|}
\hline$t \bar{t} W^{+}: \sigma(\mathrm{pb})$ & $8 \mathrm{TeV}$ & $13 \mathrm{TeV}$ & $100 \mathrm{TeV}$ \\
\hline LO QCD & $1.003 \cdot 10^{-1}$ & $2.496 \cdot 10^{-1}\left(7.749 \cdot 10^{-3}\right)$ & 3.908 \\
NLO QCD & $4.089 \cdot 10^{-2}$ & $1.250 \cdot 10^{-1}\left(4.624 \cdot 10^{-3}\right)$ & 6.114 \\
\hline LO EW & 0 & 0 & 0 \\
LO EW no $\gamma$ & 0 & 0 & 0 \\
\hline NLO EW & $-6.899 \cdot 10^{-3}$ & $-1.931 \cdot 10^{-2}\left(-1.490 \cdot 10^{-3}\right)$ & $-3.650 \cdot 10^{-1}$ \\
NLO EW no $\gamma$ & $-7.103 \cdot 10^{-3}$ & $-1.988 \cdot 10^{-2}\left(-1.546 \cdot 10^{-3}\right)$ & $-3.762 \cdot 10^{-1}$ \\
\hline HBR & $2.414 \cdot 10^{-3}$ & $9.677 \cdot 10^{-3}\left(5.743 \cdot 10^{-4}\right)$ & $8.409 \cdot 10^{-1}$ \\
\hline
\end{tabular}

Table 7. Same as in table 3, for $t \bar{t} W^{+}$production.

\begin{tabular}{|c|ccc|}
\hline$t \bar{t} W^{+}: \delta(\%)$ & $8 \mathrm{TeV}$ & $13 \mathrm{TeV}$ & $100 \mathrm{TeV}$ \\
\hline NLO QCD & $40.8_{-12.3}^{+11.2} \pm 2.9$ & $50.1_{-13.5}^{+14.2} \pm 2.4\left(59.7_{-17.7}^{+18.9} \pm 3.1\right)$ & $156.4_{-35.0}^{+38.3} \pm 2.4$ \\
\hline LO EW & 0 & 0 & 0 \\
LO EW no $\gamma$ & 0 & 0 & 0 \\
\hline NLO EW & $-6.9 \pm 0.2$ & $-7.7 \pm 0.2(-19.2 \pm 0.7)$ & $-9.3 \pm 0.2$ \\
NLO EW no $\gamma$ & $-7.1 \pm 0.2$ & $-8.0 \pm 0.2(-20.0 \pm 0.5)$ & $-9.6 \pm 0.1$ \\
\hline HBR & 2.41 & $3.88(7.41)$ & 21.52 \\
\hline
\end{tabular}

Table 8. Same as in table 4 , for $t \bar{t} W^{+}$production.

the one-loop contributions of weak origin have already been given in ref. [31]; they are not $t \bar{t} H$-specific, and hence will not be repeated here. We limit ourselves to observing, by inspection of tables $4,6,8$, and 10, that relative to the LO QCD cross sections the $t \bar{t} H$ and $t \bar{t} Z$ HBR contributions have a mild dependence on the c.m. energy (slightly increasing for the former process and decreasing for the latter one); the NLO EW contribution tend to become clearly dominant over HBR by increasing the collider energy and especially in a boosted scenario. The situation is quite the opposite for $t \bar{t} W^{ \pm}$production, where the growth of the HBR rates is not matched by that of the NLO EW terms, so that the HBR cross section is largely dominant over the latter at a $100 \mathrm{TeV}$ collider (but not quite so in a boosted configuration at the LHC Run II). The origin of this fact is the same as that responsible for the growth of the NLO QCD contributions, namely partonic luminosities; in particular, the $t \bar{t} W^{+} W^{-}$final state can be obtained from a $g g$-initiated partonic process. While the above statement must be carefully reconsidered in the context of fully-realistic simulations, where acceptance cuts are imposed on the decay products of the tops and of the vector bosons, it does say that, in such simulations, HBR contributions cannot simply be neglected. Note that the behaviour with the c.m. energy of the $t \bar{t} W^{+}$and $t \bar{t} W^{-}$cross sections is not identical, mainly owing to the fact that the former (latter) process is more sensitive to valence (sea) quark densities.

We now turn to discussing how the results presented so far might be affected by a change of EW scheme. We thus give predictions obtained in the $G_{\mu}$ scheme, with the parameters set as in eq. (3.6); we limit ourselves to considering the 13-TeV LHC, and do 


\begin{tabular}{|c|ccc|}
\hline$t \bar{t} W^{-}: \sigma(\mathrm{pb})$ & $8 \mathrm{TeV}$ & $13 \mathrm{TeV}$ & $100 \mathrm{TeV}$ \\
\hline LO QCD & $4.427 \cdot 10^{-2}$ & $1.265 \cdot 10^{-1}\left(3.186 \cdot 10^{-3}\right)$ & 2.833 \\
NLO QCD & $1.870 \cdot 10^{-2}$ & $6.515 \cdot 10^{-2}\left(2.111 \cdot 10^{-3}\right)$ & 4.351 \\
\hline LO EW & 0 & 0 & 0 \\
LO EW no $\gamma$ & 0 & 0 & 0 \\
\hline NLO EW & $-2.634 \cdot 10^{-3}$ & $-8.502 \cdot 10^{-3}\left(-5.838 \cdot 10^{-4}\right)$ & $-2.400 \cdot 10^{-1}$ \\
NLO EW no $\gamma$ & $-2.761 \cdot 10^{-3}$ & $-8.912 \cdot 10^{-3}\left(-6.094 \cdot 10^{-4}\right)$ & $-2.484 \cdot 10^{-1}$ \\
\hline HBR & $1.924 \cdot 10^{-3}$ & $8.219 \cdot 10^{-3}\left(4.781 \cdot 10^{-4}\right)$ & $8.192 \cdot 10^{-1}$ \\
\hline
\end{tabular}

Table 9. Same as in table 3 , for $t \bar{t} W^{-}$production.

\begin{tabular}{|c|ccc|}
\hline$t \bar{t} W^{-}: \delta(\%)$ & $8 \mathrm{TeV}$ & $13 \mathrm{TeV}$ & $100 \mathrm{TeV}$ \\
\hline NLO QCD & $42.2_{-12.7}^{+11.9} \pm 3.3$ & $51.5_{-13.8}^{+14.8} \pm 2.8\left(66.3_{-19.6}^{+21.7} \pm 3.9\right)$ & $153.6_{-34.9}^{+37.7} \pm 2.2$ \\
\hline LO EW & 0 & 0 & 0 \\
LO EW no $\gamma$ & 0 & 0 & 0 \\
\hline NLO EW & $-6.0 \pm 0.3$ & $-6.7 \pm 0.2(-18.3 \pm 0.8)$ & $-8.5 \pm 0.2$ \\
NLO EW no $\gamma$ & $-6.2 \pm 0.2$ & $-7.0 \pm 0.2(-19.1 \pm 0.6)$ & $-8.8 \pm 0.1$ \\
\hline HBR & 4.35 & $6.50(15.01)$ & 28.91 \\
\hline
\end{tabular}

Table 10. Same as in table 4 , for $t \bar{t} W^{-}$production.

not include HBR cross sections in this study. We define a quantity analogous to that of eq. (3.10) in the $G_{\mu}$ scheme:

$$
\delta_{\mathrm{X}}^{G_{\mu}}=\frac{\sigma_{\mathrm{X}}^{G_{\mu}}}{\sigma_{\mathrm{LO} \mathrm{QCD}}^{G_{\mu}}} .
$$

We also introduce the following ratios, that help measure the differences between analogous results in the two schemes:

$$
\begin{aligned}
\Delta_{\mathrm{LO} \mathrm{QCD}}^{G_{\mu}} & =\frac{\sigma_{\mathrm{LO} \mathrm{QCD}}-\sigma_{\mathrm{LO} \mathrm{QCD}}^{G_{\mu}}}{\sigma_{\mathrm{LO} \mathrm{QCD}}}, \\
\Delta_{\mathrm{LO} \mathrm{EW}}^{G_{\mu}} & =\frac{\sigma_{\mathrm{LO} \mathrm{QCD}}+\sigma_{\mathrm{LO} \mathrm{EW}}-\left(\sigma_{\mathrm{LO} \mathrm{QCD}}^{G_{\mu}}+\sigma_{\mathrm{LO} \mathrm{EW}}^{G_{\mu}}\right)}{\sigma_{\mathrm{LO} \mathrm{QCD}}+\sigma_{\mathrm{LO} \mathrm{EW}}}, \\
\Delta_{\mathrm{NLO} \mathrm{EW}}^{G_{\mu}} & =\frac{\sigma_{\mathrm{LO} \mathrm{QCD}}+\sigma_{\mathrm{LO} \mathrm{EW}}+\sigma_{\mathrm{NLO} \mathrm{EW}}-\left(\sigma_{\mathrm{LO} \mathrm{QCD}}^{G_{\mu}}+\sigma_{\mathrm{LO} \mathrm{EW}}^{G_{\mu}}+\sigma_{\mathrm{NLO} \mathrm{EW}}^{G_{\mu}}\right)}{\sigma_{\mathrm{LO} \mathrm{QCD}}+\sigma_{\mathrm{LO} \mathrm{EW}}+\sigma_{\mathrm{NLO} \mathrm{EW}}} .
\end{aligned}
$$

The results are collected in table 11, where for ease of comparison we also report the relevant predictions given previously in the $\alpha\left(m_{Z}\right)$ scheme (see tables $3-10$ ).

The scheme dependence of the dominant LO term, $\sigma_{\mathrm{LO} \text { QCD }}$, is solely due to the value of $\alpha$; thus, the $2.5 \%$ reported in the third row of table 11 is simply the relative difference between the two values of $\alpha$ given in eqs. (3.5) and (3.6), since this LO term factorises a single power of $\alpha$. The smallness of $\sigma_{\mathrm{LO}} \mathrm{EW}$ is such that $\Delta_{\mathrm{LO} \mathrm{EW}}^{G_{\mu}}$, defined in eq. (3.13), 


\begin{tabular}{|c|cccc|}
\hline & $t \bar{t} H$ & $t \bar{t} Z$ & $t \bar{t} W^{+}$ & $t \bar{t} W^{-}$ \\
\hline$\sigma_{\mathrm{LO} \mathrm{QCD}}(\mathrm{pb})$ & $3.617 \cdot 10^{-1}$ & $5.282 \cdot 10^{-1}$ & $2.496 \cdot 10^{-1}$ & $1.265 \cdot 10^{-1}$ \\
$\sigma_{\mathrm{LO} Q \mathrm{QCD}}^{G_{\mu}}(\mathrm{pb})$ & $3.527 \cdot 10^{-1}$ & $5.152 \cdot 10^{-1}$ & $2.433 \cdot 10^{-1}$ & $1.234 \cdot 10^{-1}$ \\
\hline$\Delta_{\mathrm{LO} \mathrm{QCD}}^{G_{\mu}}(\%)$ & 2.5 & 2.5 & 2.5 & 2.5 \\
\hline$\delta_{\mathrm{LO} \mathrm{EW}}(\%)$ & 1.2 & 0.0 & 0 & 0 \\
$\delta_{\mathrm{LO} \mathrm{EW}}^{G_{\mu}}(\%)$ & 1.2 & 0.0 & 0 & 0 \\
\hline$\Delta_{\mathrm{LO} \mathrm{EW}}^{G_{\mu}}(\%)$ & 2.5 & 2.5 & 2.5 & 2.5 \\
\hline$\delta_{\mathrm{NLO} \mathrm{EW}}(\%)$ & -1.2 & -3.8 & -7.7 & -6.7 \\
$\delta_{\mathrm{NLO} \mathrm{EW}}^{G_{\mu}}(\%)$ & 1.8 & -0.7 & -4.5 & -3.5 \\
\hline$\Delta_{\mathrm{NLO} \mathrm{EW}}^{G_{\mu}}(\%)$ & -0.5 & -0.7 & -0.9 & -0.9 \\
\hline
\end{tabular}

Table 11. Comparison between results in the $\alpha\left(m_{Z}\right)$ and $G_{\mu}$ scheme, at $13 \mathrm{TeV}$.

is largely dominated by $\sigma_{\mathrm{LO}} \mathrm{QCD}$. Hence its values are also equal to $2.5 \%$ within the numerical accuracy of our results; by increasing the statistics, one would observe tiny differences w.r.t. the predictions for $\Delta_{\mathrm{LO}}^{G_{\mu}} \mathrm{QCD}$. The predictions for the relative differences at the LO imply that a change of EW scheme may be significant, being of the same order as the NLO EW relative contributions, in particular in the case of $t \bar{t} H$ and $t \bar{t} Z$ production, and slightly less so for $t \bar{t} W^{ \pm}$production (compare $\Delta_{\mathrm{LO} \text { EW }}^{G_{\mu}}$ with $\delta_{\mathrm{NLO}} \mathrm{EW}$ ). These higherorder EW results are also affected by a change of EW scheme, as one can see by comparing the results for $\delta_{\mathrm{NLO}} \mathrm{EW}$ and for $\delta_{\mathrm{NLO} \text { EW }}^{G_{\mu}}$ in table 11, with the $G_{\mu}$ scheme responsible for a systematic shift towards positive cross sections. However, the most relevant figure of merit is actually $\Delta_{\mathrm{NLO} \text { EW }}^{G_{\mu}}$, defined in eq. (3.14), which must be compared with its LO counterparts, $\Delta_{\mathrm{LO} \mathrm{QCD}}^{G_{\mu}}$ and $\Delta_{\mathrm{LO} \text { EW }}^{G_{\mu}}$; the values of the former ratio are significantly smaller than those of the latter two ratios, as a result of the stabilisation against changes of scheme that is characteristic of higher-order computations.

We conclude this section by mentioning that we have also computed the LO contributions of $\mathcal{O}\left(\alpha^{3}\right)$ to the total rates, since they factor the same power of $\lambda^{6}$ as the $\mathcal{O}\left(\alpha_{S}^{2} \alpha^{2}\right)$ NLO terms, according to the naive scaling law $\alpha_{S} \rightarrow \lambda \alpha_{S}$ and $\alpha \rightarrow \lambda^{2} \alpha$. We find that these third-leading LO rates are smaller (for $t \bar{t} H$ and $t \bar{t} Z$ ), or much smaller (for $t \bar{t} W^{ \pm}$, by a factor of about ten), than the NLO EW ones; furthermore, they are not enhanced by any Sudakov logarithms at large hardness. We finally remark that photon-initiated contributions of $\mathcal{O}\left(\alpha^{3}\right)$ are negligibly small. For these reasons, we have not reported any $\mathcal{O}\left(\alpha^{3}\right)$ results in the tables above, and have ignored their contributions to differential distributions.

\subsection{Differential distributions}

In analogy with ref. [31], we have considered the following observables:

- the transverse momentum of the heavy boson $p_{T}(V)$;

- the transverse momentum of the top quark $p_{T}(t)$; 
- the transverse momentum of the top-antitop pair $p_{T}(t \bar{t})$;

- the invariant mass of the top-antitop-heavy boson system $M(t \bar{t} V)$;

- the rapidity of the top quark $y(t)$;

- the rapidity separation between the top-antitop pair and the heavy boson $\Delta y(t \bar{t}, V)$.

We present these six observables for each of the four production processes at the $13-\mathrm{TeV}$ LHC, without and with the cuts of eq. (3.1): $t \bar{t} H$ in figures 1 and $2, t \bar{t} Z$ in figures 3 and $4, t \bar{t} W^{+}$in figures 5 and 6 , and $t \bar{t} W^{-}$in figures 7 and 8 , respectively. We use an identical layout for all the plots, with a main frame and three insets; we employ the labelling convention introduced in table 1, and used in section 3.1 for the total rates. Four histograms appear in the main frame, that represent the differential cross sections; they are the predictions for LO QCD (dashed black), LO QCD + NLO QCD (solid red overlayed with full circles), and LO QCD + NLO QCD + LO EW + NLO EW (solid blue and green diamonds, with and without photon density respectively). In the upper and middle insets, the bin-by-bin ratios of the latter three histograms over the first one (i.e. LO QCD) are presented, by using the same patterns as in the main frame. The upper insets also display a grey band, centered around the LO QCD + NLO QCD prediction, which represents the fractional scale variation of this cross section. Conversely, the middle insets show the fractional PDF uncertainties that affect the full NLO cross section LO QCD+NLO QCD+ LO EW + NLO EW: with (blue band) and without (green error symbols) photon density. Finally, in the bottom insets we present the ratios of the following three quantities over LO QCD: NLO QCD (red solid), LO EW + NLO EW (solid blue with photon density, and green diamonds without photon density), and HBR (purple dot-dashed). PDF-uncertainty bands for the relative LO EW +NLO EW predictions are also given, with the same patterns as their analogues in the middle insets. Note that HBR processes that feature two identical vector bosons in the final state might give up to two contributions per event to the $p_{T}(V)$, $M(t \bar{t} V)$, and $\Delta y(t \bar{t}, V)$ distributions.

The general features of these differential results are largely independent of the specific process considered. When no cuts are applied (figures 1, 3, 5, and 7), regions close to threshold (i.e. associated with small transverse momenta) are dominated by QCD contributions; by adding EW effects one generally shifts downwards the cross sections, but still within the theoretical uncertainties. By moving towards large $p_{T}$ 's, the $K$ factors due to the leading NLO term tend to increase, for some observables and processes in a truly dramatic manner. The relative size of EW contributions also increases in absolute value in the same regions; since the corresponding cross sections are negative, this partly compensates the growth induced by QCD terms. Furthermore, at variance with what happens at threshold, such a compensation is quite often significant, being of the same order as, or larger than, the theoretical uncertainty; the largest effects are seen in $t \bar{t} W^{ \pm}$production. This fact, together with the observation that $K$ factors are not flat, implies that both QCD and EW higher-order effects need to be taken into account at a fully differential level for a precision study of $t \bar{t} V$ production. As was already observed in the case of total rates, 
the impact of photon-initiated processes is not large on NLO-accurate results; there is a slight fractional increase when moving towards large transverse momenta, but given the current theoretical uncertainties this is hardly significant; a similar conclusion applies to the impact of the photon density uncertainty on the overall PDF errors. The largest effects are seen in $t \bar{t} H$ production. A notable exception to these statements is to be found at large top-rapidity values in $t \bar{t} H$ and $t \bar{t} Z$ production, where the cross sections with or without the photon-initiated processes exhibit large differences. However, such differences are offset by a very significant increase of the PDF uncertainty, which is driven by the poorly known photon density.

Given these results, it is not surprising that the various higher-order effects are enhanced when one imposes the cuts of eq. (3.1) - see figures $2,4,6$, and 8. In this case, the importance of taking into account EW effects is obvious; this includes the HBR cross sections which, according to our results, are particularly large for certain observables in $t \bar{t} W^{ \pm}$ production, in keeping with what has been already found for total rates. The sharp thresholds in $p_{T}(t \bar{t})$ and $M(t \bar{t} V)$, and the knee at $p_{T}(t) \sim 400 \mathrm{GeV}$, are LO features common to all processes, which become less dramatic when NLO corrections are included. Their origins have been discussed in ref. [31] (for $t \bar{t} H$ production, but those arguments apply to $t \bar{t} Z$ and $t \bar{t} W^{ \pm}$production as well), and therefore will not be repeated here.

\section{Conclusions and outlook}

We have studied the production of a $t \bar{t}$ pair in association with a heavy SM boson (Higgs, $Z$, or $\left.W^{ \pm}\right)$at a $p p$ collider with three different c.m. energies $(8,13$, and $100 \mathrm{TeV})$. Our predictions are obtained by computing the two dominant terms at both the leading and the next-to-leading order in a mixed perturbative expansion in the QCD $\left(\alpha_{S}\right)$ and EW $(\alpha)$ couplings. Such terms factorise the coupling combinations $\alpha_{S}^{2} \alpha$ and $\alpha_{S} \alpha^{2}$ at the LO, and $\alpha_{S}^{3} \alpha$ and $\alpha_{S}^{2} \alpha^{2}$ at the NLO; the latter two contributions are usually denoted as QCD and EW NLO corrections, respectively. The $\mathcal{O}\left(\alpha_{S}^{2} \alpha^{2}\right)$ results for $t \bar{t} H$ production had been previously presented in the literature in refs. $[31,32]$ (with the former paper ignoring QED effects); those for $t \bar{t} Z, t \bar{t} W^{+}$, and $t \bar{t} W^{-}$are given here for the first time.

These $t \bar{t} V$ processes are characterised by tiny cross sections, the total rates being smaller than $1 \mathrm{pb}$ at LHC energies, and of the order of $10 \mathrm{pb}$ at a $100-\mathrm{TeV}$ collider. However, in view of the luminosity foreseen at the LHC Run II and at future colliders, it will become possible to measure them with a good accuracy; furthermore, $t \bar{t} Z$ and $t \bar{t} W^{ \pm}$are significant backgrounds to several BSM searches, that typically feature multilepton final states. There are thus compelling phenomenological motivations to increase the precision of the theoretical predictions for $t \bar{t} V$ production, which is what we have done in this paper. From a technical viewpoint, our calculations have been performed with MadGraph5_AMC@NLO [33], and are fully automated. They are the first public results obtained with such a code that include QED subtractions, and they constitute part of the validation procedure that will lead to the public release of an upgraded MADGRAPH5_AMC@NLO capable of handling mixed-coupling expansions at the NLO. We point out that no part of the code has been specifically constructed or modified in order to handle $t \bar{t} V$ production. 

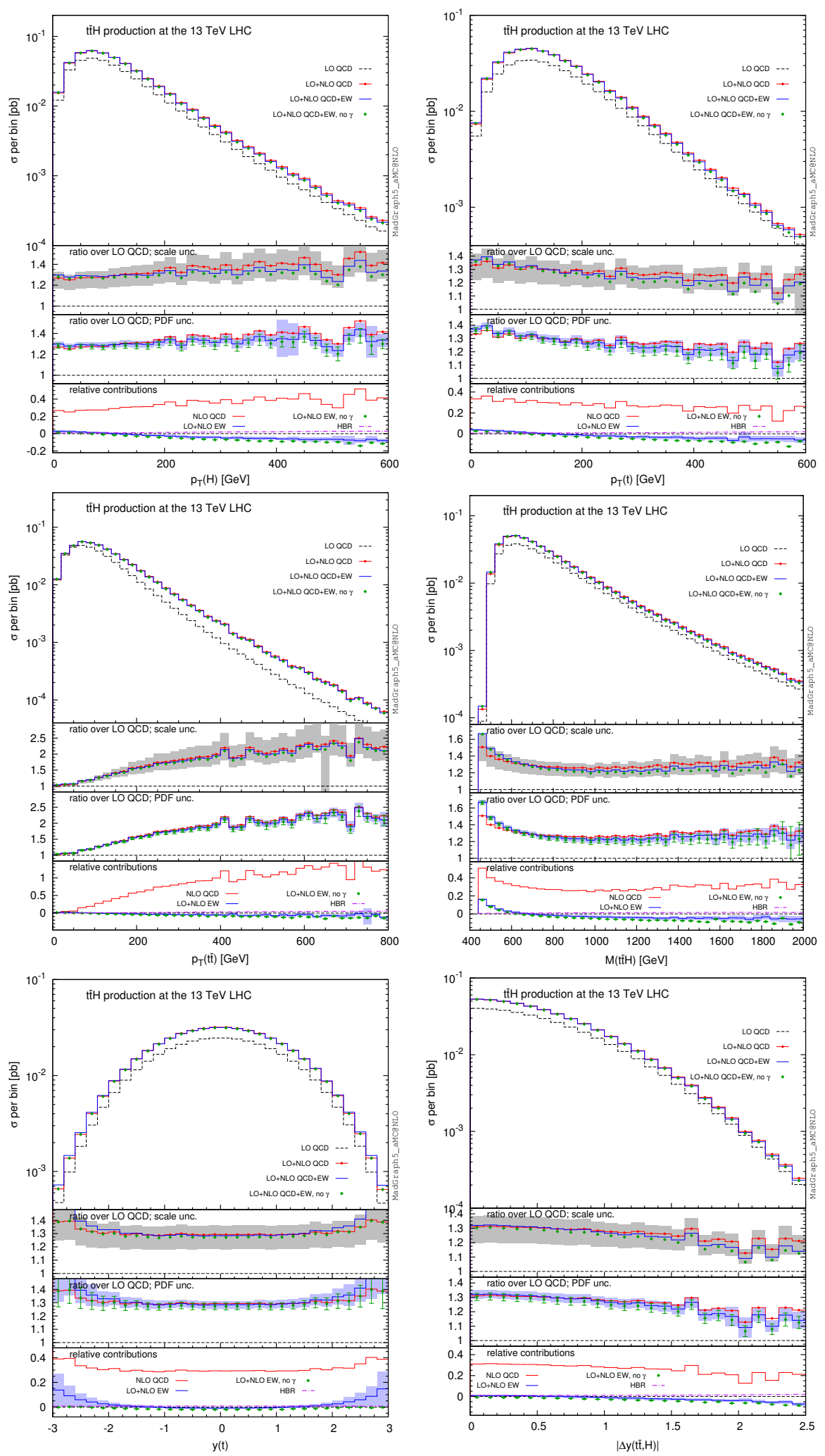

Figure 1. LO- and NLO-accurate results for $t \bar{t} H$ production at $13 \mathrm{TeV}$. 

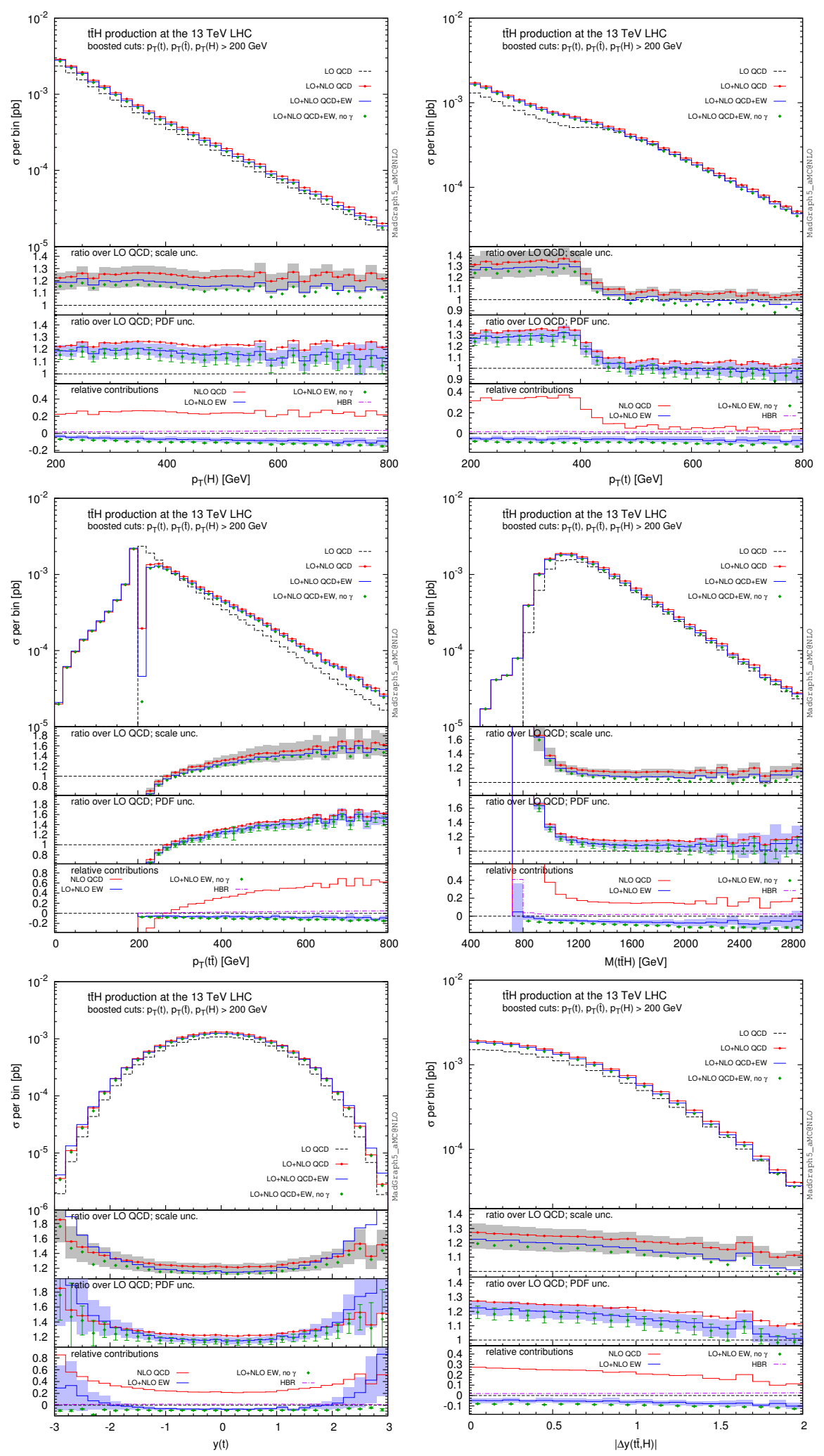

Figure 2. Same as in figure 1, with the cuts of eq. (3.1). 

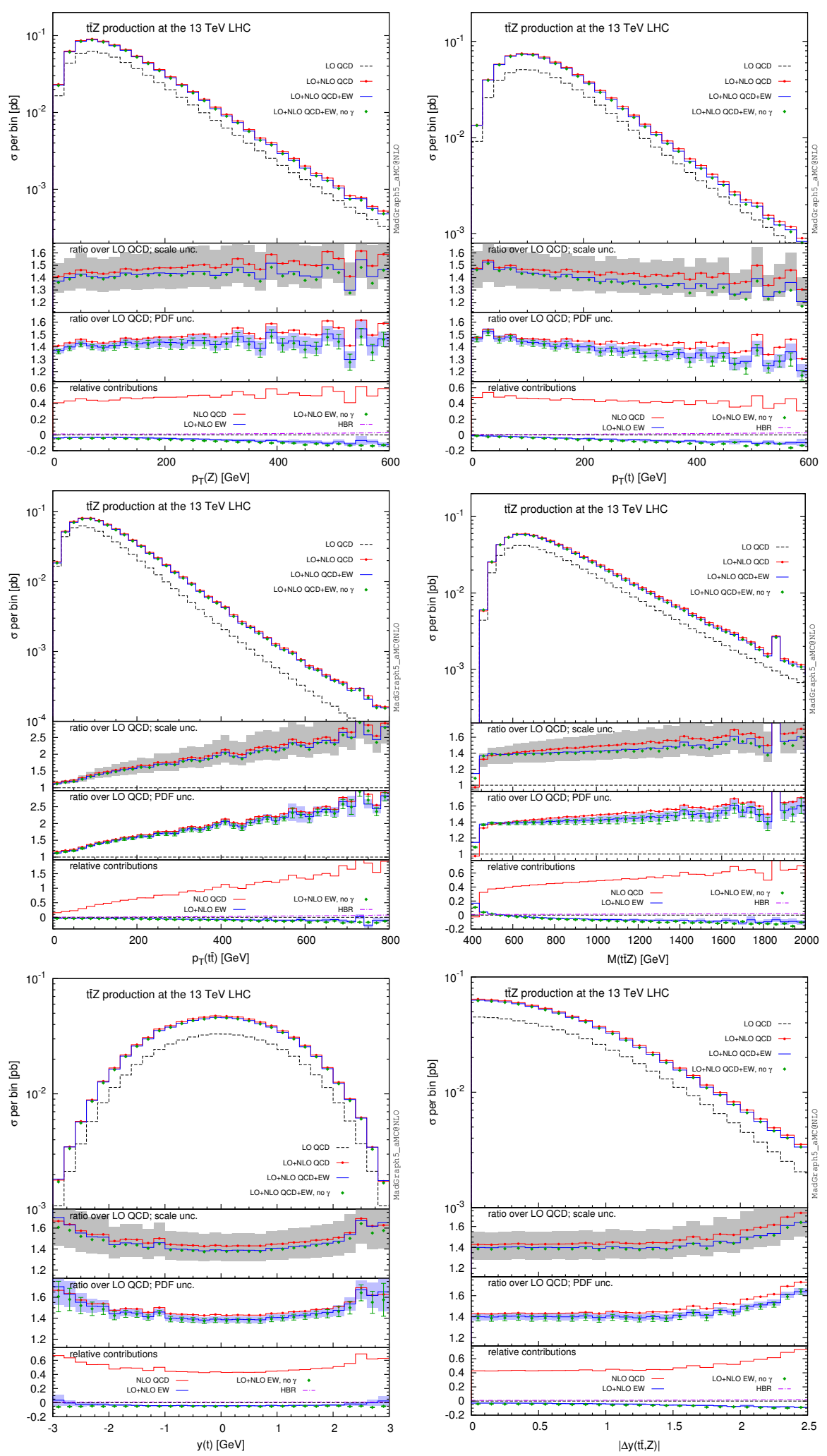

Figure 3. Same as in figure 1 , for $t \bar{t} Z$ production. 

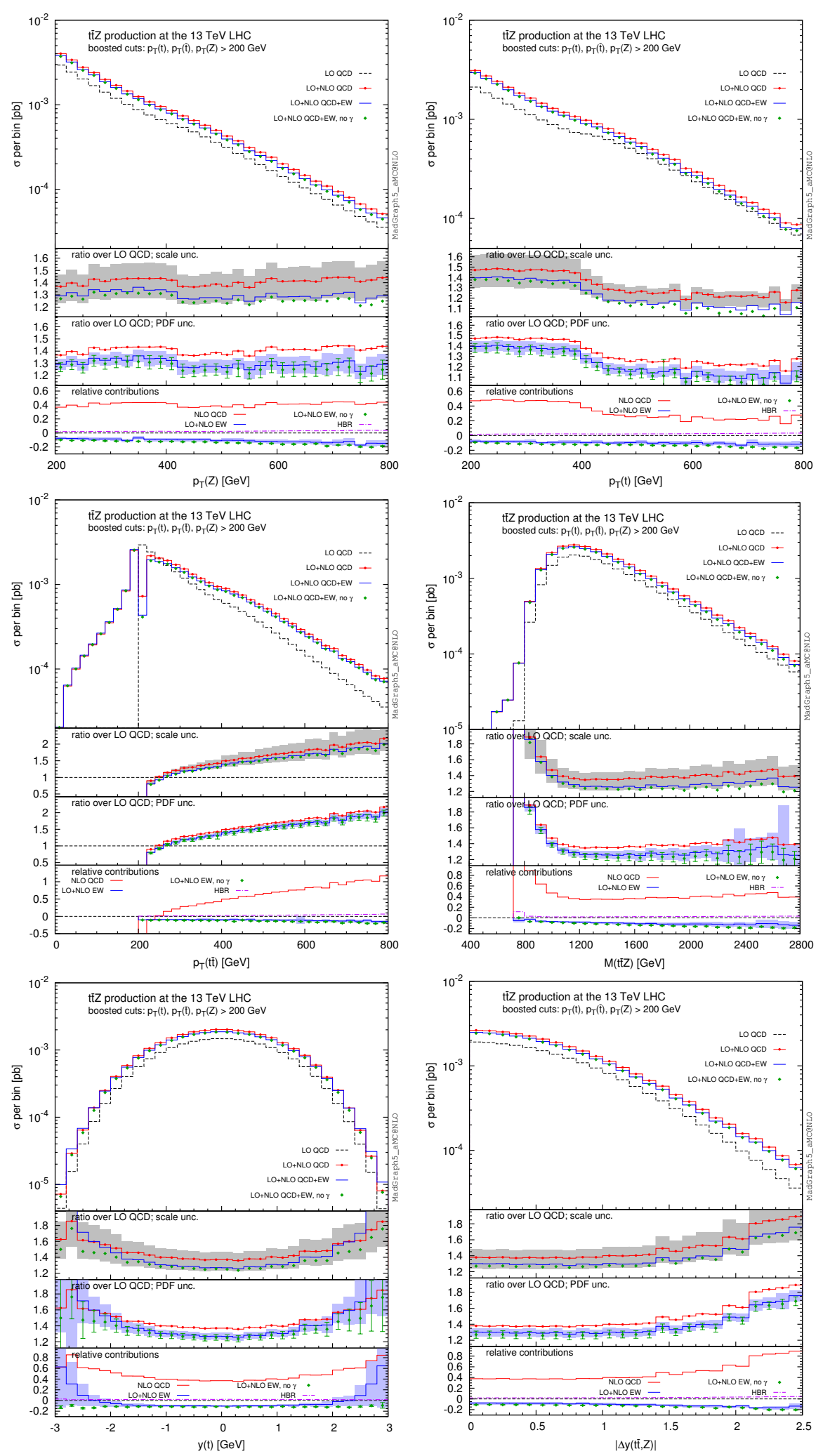

Figure 4. Same as in figure 2, for $t \bar{t} Z$ production. 

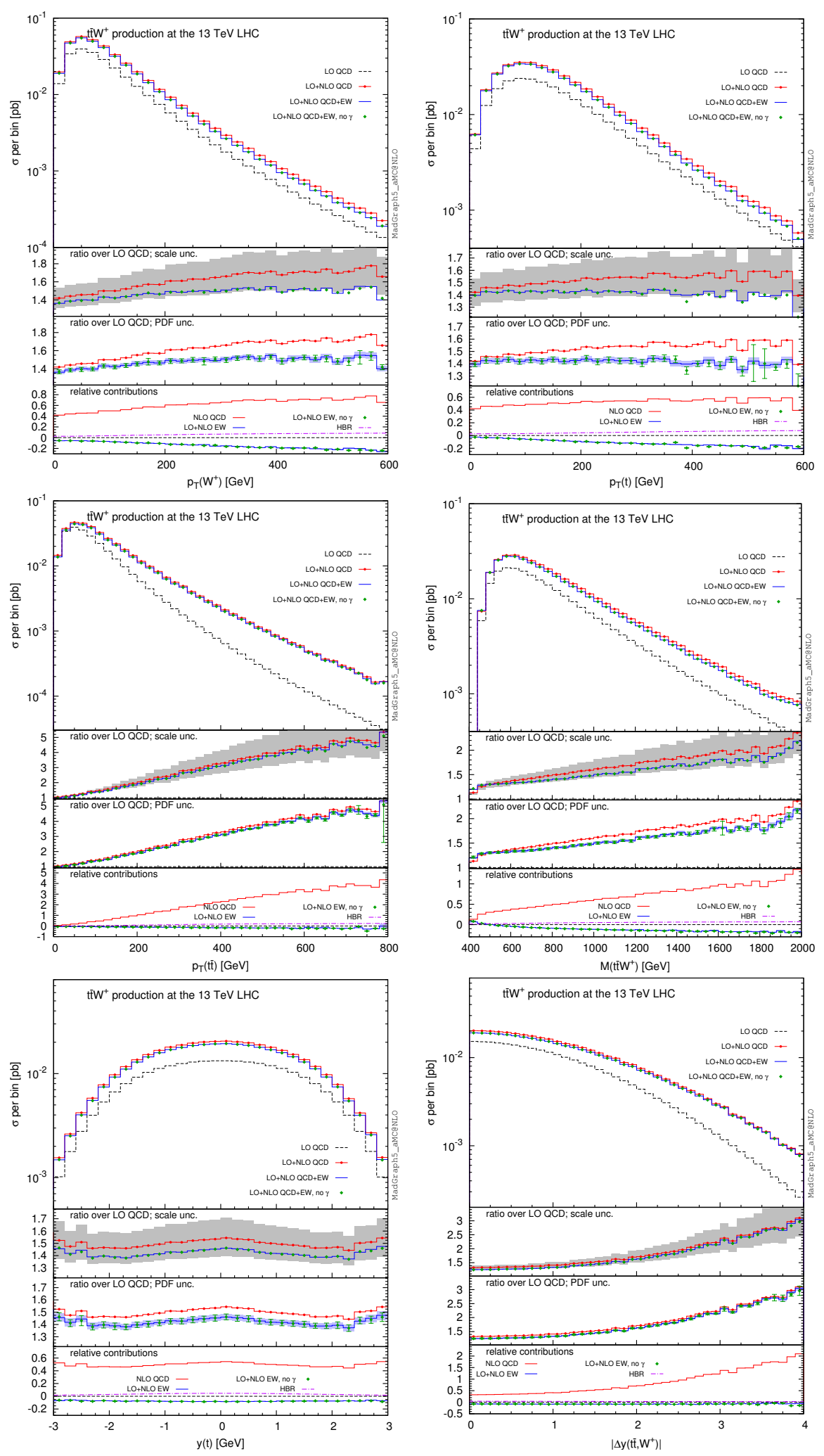

Figure 5. Same as in figure 1, for $t \bar{t} W^{+}$production. 

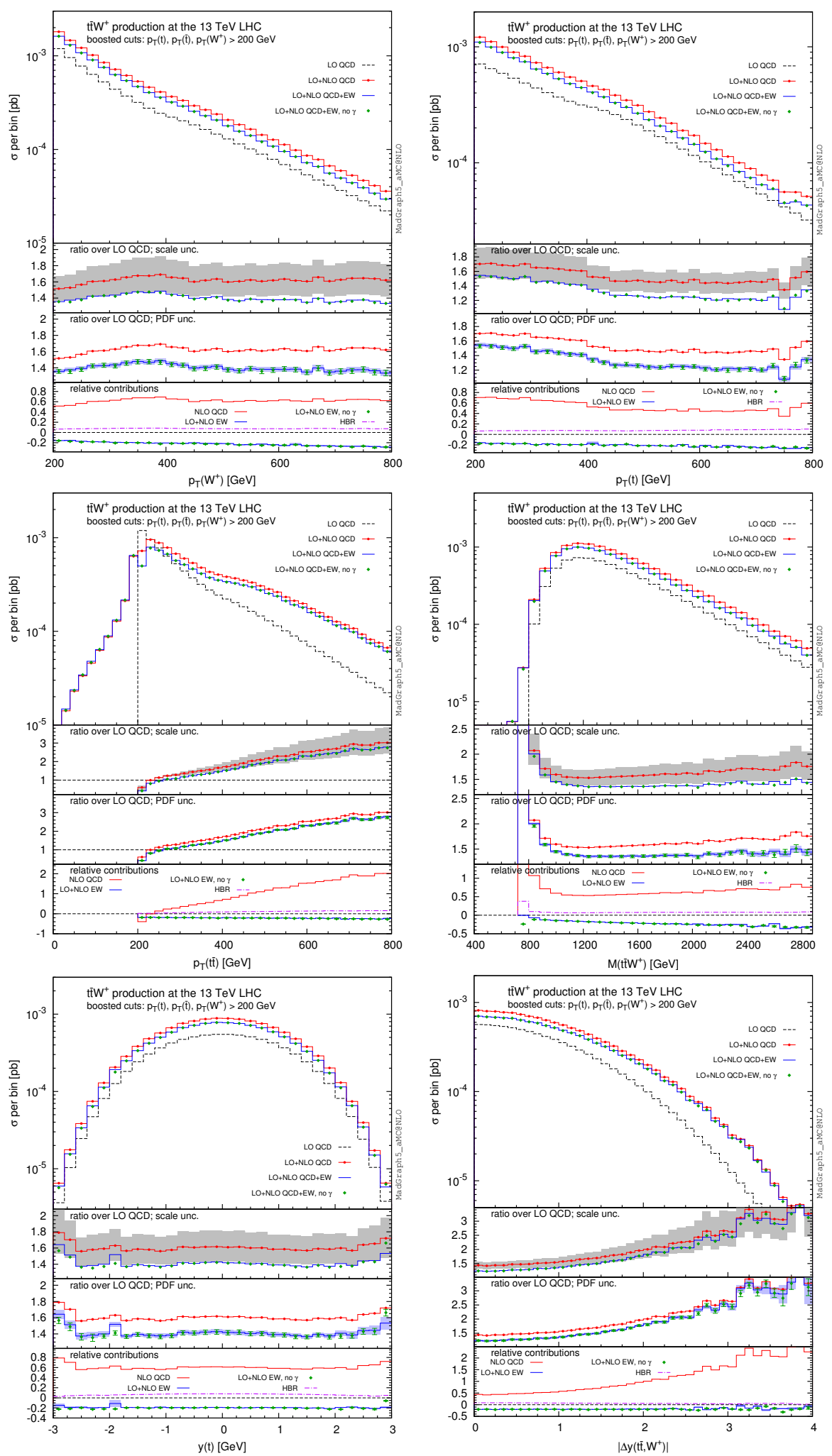

Figure 6. Same as in figure 2 , for $t \bar{t} W^{+}$production. 

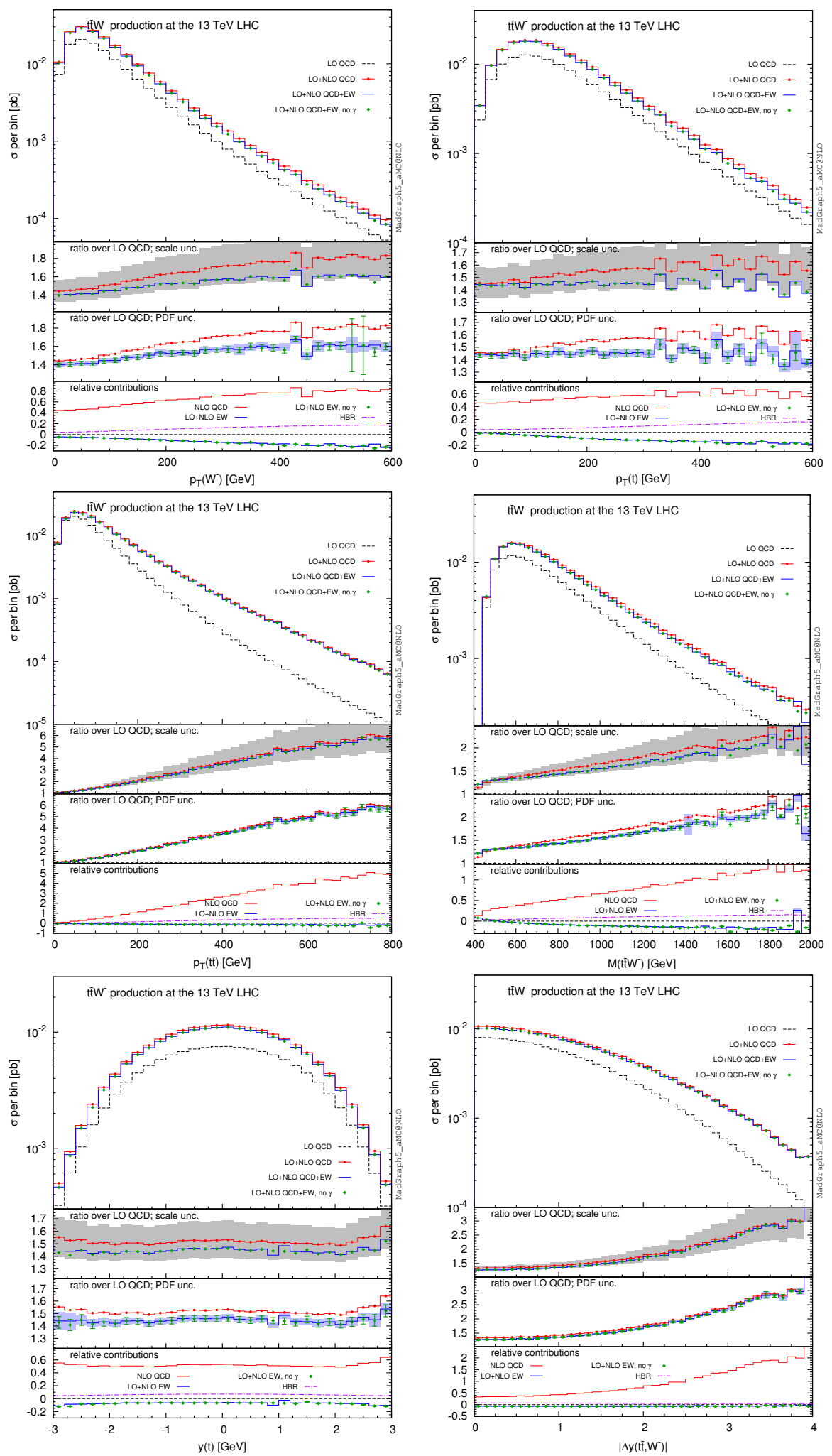

Figure 7. Same as in figure 1, for $t \bar{t} W^{-}$production. 

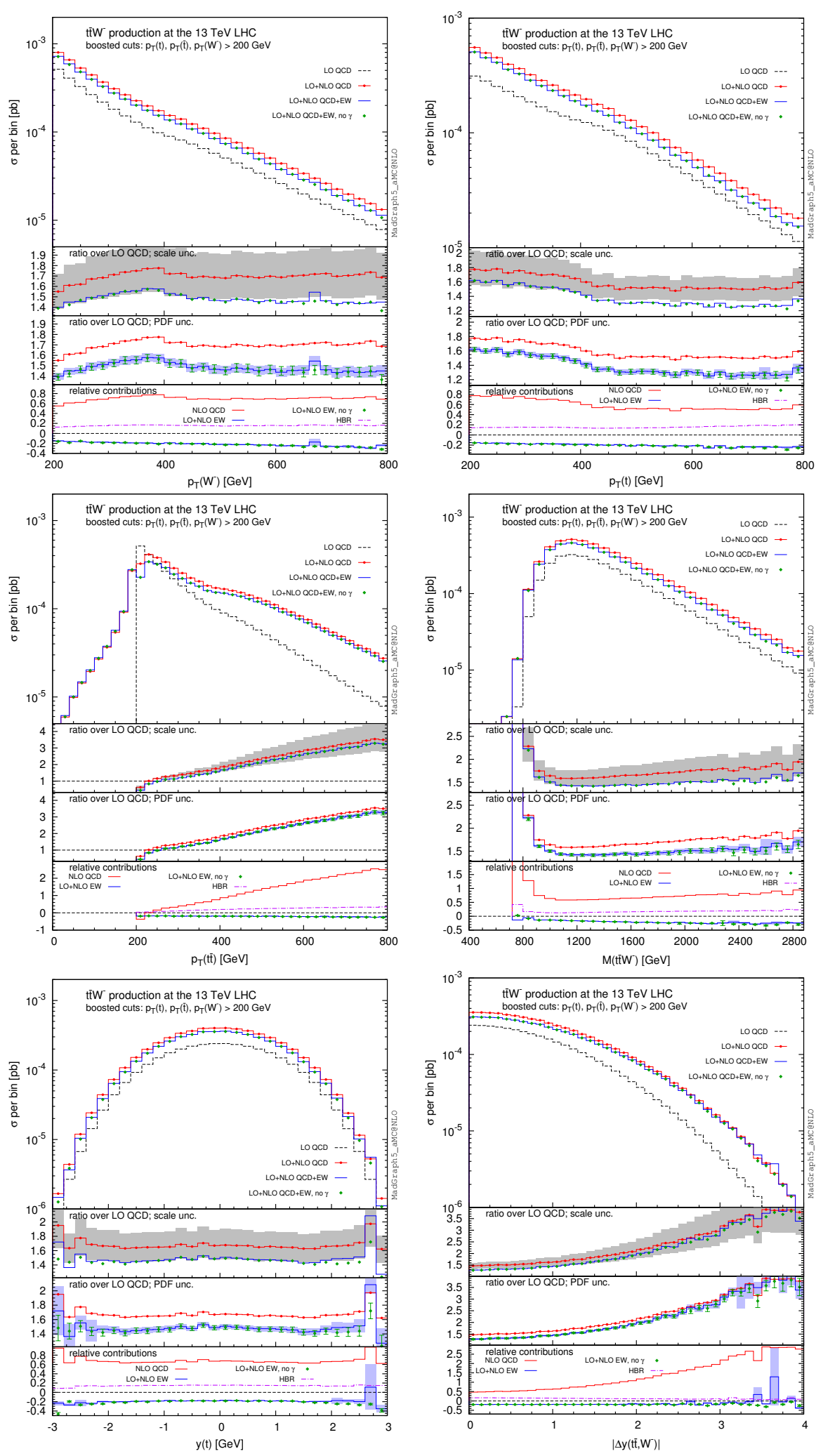

Figure 8. Same as in figure 2, for $t \bar{t} W^{-}$production. 
The main findings of our study are the following. The fully differential computation of higher-order corrections is essential in order to attain a realistic description of the processes at hand. $K$ factors are large and not flat, and tend to grow with the collider c.m. energy; such a growth is particularly spectacular in the case of $t \bar{t} W^{ \pm}$production, owing to the impact of gluon-initiated partonic processes which are absent at the LO (at variance with what happens for $t \bar{t} H$ and $t \bar{t} Z$ ). At a given collider energy, a similar feature is observed when at least one of the final-state particles has a large transverse momentum. Theoretical uncertainties are predominantly due to scale variations; PDF errors are smaller but not negligible. For the same reason as explained above, scale uncertainties become very significant in $t \bar{t} W^{ \pm}$production at high energies and/or transverse momenta. The considerable size of higher-order corrections stems predominantly from $\mathcal{O}\left(\alpha_{S}^{3} \alpha\right)$ terms. However, effects of EW origin do change the pure-QCD results in a way which is significant (i.e. of the same order as, or larger than, the theoretical uncertainties) at large energies and $p_{T}$ 's. The precise impact of such effects depends on the observable (and, of course, on the process), which implies again the necessity of performing one's calculations in a fully differential manner. QED corrections lead to the inclusion of processes with initial-state photons. We find that these give a modest fractional contribution to the NLO $\mathcal{O}\left(\alpha_{S}^{2} \alpha^{2}\right)$ term, but a very large one to the $\mathrm{LO} \mathcal{O}\left(\alpha_{S} \alpha^{2}\right)$ term (for $t \bar{t} H$ and $t \bar{t} Z$, being identically zero in $t \bar{t} W^{ \pm}$production); however, the latter does not induce a visible change in the physical NLO-accurate cross section, since the corresponding contribution is quite small in absolute value. These facts imply that, although the large uncertainties on the photon PDF are sizable when one only considers second-leading LO and NLO terms, they essentially become irrelevant as far as the overall uncertainties on the NLO-accurate results are concerned (with the exception of large top rapidities in $t \bar{t} H$ and $t \bar{t} Z$ production). Finally, we find that processes with a $t \bar{t}$ and a heavy-boson pair in the final state, which we have called HBR, might be responsible for detectable effects, particularly in the case of $t \bar{t} W^{ \pm}$production: a definite conclusion on this point can only be reached with a realistic acceptance study (either by including HBR contributions in the $t \bar{t} V$ cross section inclusive in $V$, or by subtracting them if the $t \bar{t} V$ cross section is exclusive in a single $V$ ).

\section{Acknowledgments}

We are grateful to J. Rojo for clarifications concerning NNPDF2.3QED and for comments on the manuscript, to S. Uccirati for producing pointwise results with RECOLA for crosschecks, and to R. Frederix, F. Maltoni, and M. Mangano for discussions and collaboration at different stages of this work. This work is supported in part (DP and HSS) by, and performed in the framework of, the ERC grant 291377 "LHCtheory: Theoretical predictions and analyses of LHC physics: advancing the precision frontier". The work of VH is supported by the SNF with grant PBELP2 146525. The work of MZ is supported by the ERC grant "Higgs@LHC", in part by the Research Executive Agency (REA) of the European Union under the Grant Agreement number PITN-GA-2010-264564 (LHCPhenoNet), and in part by the ILP LABEX (ANR-10-LABX-63), in turn supported by French state funds managed by the ANR within the "Investissements d'Avenir" programme under reference ANR-11-IDEX-0004-02. 
Open Access. This article is distributed under the terms of the Creative Commons Attribution License (CC-BY 4.0), which permits any use, distribution and reproduction in any medium, provided the original author(s) and source are credited.

\section{References}

[1] R.M. Barnett, J.F. Gunion and H.E. Haber, Discovering supersymmetry with like sign dileptons, Phys. Lett. B 315 (1993) 349 [hep-ph/9306204] [INSPIRE].

[2] M. Guchait and D.P. Roy, Like sign dilepton signature for gluino production at CERN LHC including top quark and Higgs boson effects, Phys. Rev. D 52 (1995) 133 [hep-ph/9412329] [INSPIRE].

[3] H. Baer, C.-h. Chen, F. Paige and X. Tata, Signals for minimal supergravity at the CERN large hadron collider. 2: Multi-lepton channels, Phys. Rev. D 53 (1996) 6241 [hep-ph/9512383] [INSPIRE].

[4] H.-C. Cheng, K.T. Matchev and M. Schmaltz, Bosonic supersymmetry? Getting fooled at the CERN LHC, Phys. Rev. D 66 (2002) 056006 [hep-ph/0205314] [InSPIRE].

[5] R. Contino and G. Servant, Discovering the top partners at the LHC using same-sign dilepton final states, JHEP 06 (2008) 026 [arXiv:0801.1679] [INSPIRE].

[6] ATLAS collaboration, Search for $t \bar{t} Z$ production in the three lepton final state with $4.7 \mathrm{fb}^{-1}$ of $\sqrt{s}=7$ TeV pp collision data collected by the ATLAS detector, ATLAS-CONF-2012-126 (2012).

[7] ATLAS collaboration, Evidence for the associated production of a $W$ boson and a top quark in ATLAS at $\sqrt{s}=7 \mathrm{TeV}$, Phys. Lett. B 716 (2012) 142 [arXiv:1205.5764] [INSPIRE].

[8] CMS collaboration, Measurement of associated production of vector bosons and top quark-antiquark pairs at $\sqrt{s}=7 \mathrm{TeV}$, Phys. Rev. Lett. 110 (2013) 172002 [arXiv:1303.3239] [INSPIRE].

[9] CMS collaboration, Measurement of top quark-antiquark pair production in association with a $W$ or $Z$ boson in pp collisions at $\sqrt{s}=8$ TeV, Eur. Phys. J. C 74 (2014) 3060 [arXiv:1406.7830] [INSPIRE].

[10] ATLAS collaboration, Evidence for the associated production of a vector boson ( $W, Z$ ) and top quark pair in the dilepton and trilepton channels in pp collision data at $\sqrt{s}=8 \mathrm{TeV}$ collected by the ATLAS detector at the LHC, ATLAS-CONF-2014-038 (2014).

[11] ATLAS collaboration, Search for the Standard Model Higgs boson produced in association with top quarks in proton-proton collisions at $\sqrt{s}=7 \mathrm{TeV}$ using the ATLAS detector, ATLAS-CONF-2012-135 (2012).

[12] ATLAS collaboration, Search for ttH production in the $H \rightarrow$ yy channel at $\sqrt{s}=8 \mathrm{TeV}$ with the ATLAS detector, ATLAS-CONF-2013-080 (2013).

[13] CMS collaboration, Search for Higgs boson production in association with top quark pairs in pp collisions, CMS-PAS-HIG-12-025.

[14] CMS collaboration, Search for $t t H$ production in events where $H$ decays to photons at $8 \mathrm{TeV}$ collisions, CMS-PAS-HIG-13-015.

[15] CMS collaboration, Search for the standard model Higgs boson produced in association with top quarks in multilepton final states, CMS-PAS-HIG-13-020. 
[16] CMS collaboration, Search for Higgs Boson Production in Association with a Top-Quark Pair and Decaying to Bottom Quarks or Tau Leptons, CMS-PAS-HIG-13-019.

[17] ATLAS collaboration, Search for $H \rightarrow \gamma \gamma$ produced in association with top quarks and constraints on the Yukawa coupling between the top quark and the Higgs boson using data taken at $7 \mathrm{TeV}$ and $8 \mathrm{TeV}$ with the ATLAS detector, Phys. Lett. B 740 (2015) 222 [arXiv: 1409.3122] [INSPIRE].

[18] CMS collaboration, Search for a standard model Higgs boson produced in association with a top-quark pair and decaying to bottom quarks using a matrix element method, Eur. Phys. J. C 75 (2015) 251 [arXiv:1502.02485] [INSPIRE].

[19] A. Lazopoulos, T. McElmurry, K. Melnikov and F. Petriello, Next-to-leading order QCD corrections to $t \bar{t} Z$ production at the LHC, Phys. Lett. B 666 (2008) 62 [arXiv:0804.2220] [INSPIRE].

[20] V. Hirschi et al., Automation of one-loop QCD corrections, JHEP 05 (2011) 044 [arXiv: 1103.0621] [INSPIRE].

[21] M.V. Garzelli, A. Kardos, C.G. Papadopoulos and Z. Trócsányi, Z0-boson production in association with a top $t \bar{t}$ pair at NLO accuracy with parton shower effects, Phys. Rev. D 85 (2012) 074022 [arXiv: 1111.1444] [INSPIRE].

[22] A. Kardos, Z. Trócsányi and C. Papadopoulos, Top quark pair production in association with a Z-boson at NLO accuracy, Phys. Rev. D 85 (2012) 054015 [arXiv:1111.0610] [InSPIRE].

[23] M.V. Garzelli, A. Kardos, C.G. Papadopoulos and Z. Trócsányi, $t \bar{t} W^{+-}$and $t \bar{t} Z$ Hadroproduction at NLO accuracy in QCD with Parton Shower and Hadronization effects, JHEP 11 (2012) 056 [arXiv:1208.2665] [INSPIRE].

[24] J.M. Campbell and R.K. Ellis, $t \bar{t} W^{+-}$production and decay at NLO, JHEP 07 (2012) 052 [arXiv: 1204.5678] [INSPIRE].

[25] W. Beenakker et al., Higgs radiation off top quarks at the Tevatron and the LHC, Phys. Rev. Lett. 87 (2001) 201805 [hep-ph/0107081] [INSPIRE].

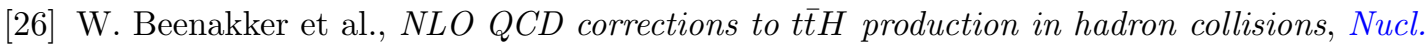
Phys. B 653 (2003) 151 [hep-ph/0211352] [INSPIRE].

[27] S. Dawson, L.H. Orr, L. Reina and D. Wackeroth, Associated top quark Higgs boson production at the LHC, Phys. Rev. D 67 (2003) 071503 [hep-ph/0211438] [INSPIRE].

[28] S. Dawson, C. Jackson, L.H. Orr, L. Reina and D. Wackeroth, Associated Higgs production with top quarks at the large hadron collider: NLO QCD corrections, Phys. Rev. D 68 (2003) 034022 [hep-ph/0305087] [INSPIRE].

[29] R. Frederix, S. Frixione, V. Hirschi, F. Maltoni, R. Pittau and P. Torrielli, Scalar and pseudoscalar Higgs production in association with a top-antitop pair, Phys. Lett. $\mathbf{B} 701$ (2011) 427 [arXiv:1104.5613] [INSPIRE].

[30] M.V. Garzelli, A. Kardos, C.G. Papadopoulos and Z. Trócsányi, Standard Model Higgs boson production in association with a top anti-top pair at NLO with parton showering, Europhys. Lett. 96 (2011) 11001 [arXiv:1108.0387] [INSPIRE].

[31] S. Frixione, V. Hirschi, D. Pagani, H.S. Shao and M. Zaro, Weak corrections to Higgs hadroproduction in association with a top-quark pair, JHEP 09 (2014) 065 [arXiv: 1407.0823] [INSPIRE]. 
[32] Y. Zhang, W.-G. Ma, R.-Y. Zhang, C. Chen and L. Guo, $Q C D N L O$ and $E W N L O$ corrections to t $\bar{t} H$ production with top quark decays at hadron collider, Phys. Lett. B 738 (2014) 1 [arXiv:1407.1110] [INSPIRE].

[33] J. Alwall et al., The automated computation of tree-level and next-to-leading order differential cross sections and their matching to parton shower simulations, JHEP 07 (2014) 079 [arXiv: 1405.0301] [INSPIRE].

[34] S. Actis, A. Denner, L. Hofer, A. Scharf and S. Uccirati, Recursive generation of one-loop amplitudes in the Standard Model, JHEP 04 (2013) 037 [arXiv:1211.6316] [INSPIRE].

[35] A. Denner, L. Hofer, A. Scharf and S. Uccirati, Electroweak corrections to lepton pair production in association with two hard jets at the LHC, JHEP 01 (2015) 094 [arXiv: 1411.0916] [INSPIRE].

[36] A. Denner, S. Dittmaier, M. Hecht and C. Pasold, NLO QCD and electroweak corrections to $W+\gamma$ production with leptonic $W$-boson decays, JHEP 04 (2015) 018 [arXiv:1412.7421] [INSPIRE].

[37] S. Kallweit, J.M. Lindert, P. Maierhöfer, S. Pozzorini and M. Schönherr, NLO electroweak automation and precise predictions for W+multijet production at the LHC, JHEP 04 (2015) 012 [arXiv: 1412.5157] [INSPIRE].

[38] NNPDF collaboration, R.D. Ball et al., Parton distributions with QED corrections, Nucl. Phys. B 877 (2013) 290 [arXiv:1308.0598] [InSPIRE].

[39] S. Frixione, Z. Kunszt and A. Signer, Three jet cross-sections to next-to-leading order, Nucl. Phys. B 467 (1996) 399 [hep-ph/9512328] [INSPIRE].

[40] S. Frixione, A general approach to jet cross-sections in QCD, Nucl. Phys. B 507 (1997) 295 [hep-ph/9706545] [INSPIRE].

[41] R. Frederix, S. Frixione, F. Maltoni and T. Stelzer, Automation of next-to-leading order computations in QCD: The FKS subtraction, JHEP 10 (2009) 003 [arXiv:0908.4272] [INSPIRE].

[42] G. Ossola, C.G. Papadopoulos and R. Pittau, Reducing full one-loop amplitudes to scalar integrals at the integrand level, Nucl. Phys. B 763 (2007) 147 [hep-ph/0609007] [INSPIRE].

[43] G. Passarino and M.J.G. Veltman, One Loop Corrections for $e^{+} e^{-}$Annihilation Into $\mu^{+} \mu^{-}$ in the Weinberg Model, Nucl. Phys. B 160 (1979) 151 [InSPIRE].

[44] A.I. Davydychev, A simple formula for reducing Feynman diagrams to scalar integrals, Phys. Lett. B 263 (1991) 107 [INSPIRE].

[45] G. Ossola, C.G. Papadopoulos and R. Pittau, CutTools: A program implementing the OPP reduction method to compute one-loop amplitudes, JHEP 03 (2008) 042 [arXiv:0711.3596] [INSPIRE].

[46] H.-S. Shao, Iregi user manual, unpublished.

[47] F. Cascioli, P. Maierhofer and S. Pozzorini, Scattering Amplitudes with Open Loops, Phys. Rev. Lett. 108 (2012) 111601 [arXiv:1111.5206] [INSPIRE].

[48] J.M. Butterworth, A.R. Davison, M. Rubin and G.P. Salam, Jet substructure as a new Higgs search channel at the LHC, Phys. Rev. Lett. 100 (2008) 242001 [arXiv:0802.2470] [INSPIRE]. 
[49] T. Plehn, G.P. Salam and M. Spannowsky, Fat Jets for a Light Higgs, Phys. Rev. Lett. 104 (2010) 111801 [arXiv:0910.5472] [INSPIRE].

[50] M.R. Buckley, T. Plehn, T. Schell and M. Takeuchi, Buckets of Higgs and Tops, JHEP 02 (2014) 130 [arXiv:1310.6034] [INSPIRE].

[51] P. Ciafaloni and D. Comelli, Sudakov enhancement of electroweak corrections, Phys. Lett. B 446 (1999) 278 [hep-ph/9809321] [INSPIRE].

[52] M. Ciafaloni, P. Ciafaloni and D. Comelli, Bloch-Nordsieck violating electroweak corrections to inclusive TeV scale hard processes, Phys. Rev. Lett. 84 (2000) 4810 [hep-ph/0001142] [INSPIRE].

[53] A. Denner and S. Pozzorini, One loop leading logarithms in electroweak radiative corrections. 1. Results, Eur. Phys. J. C 18 (2001) 461 [hep-ph/0010201] [INSPIRE].

[54] A. Denner and S. Pozzorini, One loop leading logarithms in electroweak radiative corrections. 2. Factorization of collinear singularities, Eur. Phys. J. C 21 (2001) 63 [hep-ph/0104127] [INSPIRE].

[55] C. Degrande et al., UFO - The Universal FeynRules Output, Comput. Phys. Commun. 183 (2012) 1201 [arXiv: 1108.2040] [INSPIRE].

[56] R. Frederix et al., Four-lepton production at hadron colliders: aMC@NLO predictions with theoretical uncertainties, JHEP 02 (2012) 099 [arXiv:1110.4738] [INSPIRE].

[57] NNPDF collaboration, R.D. Ball et al., A determination of parton distributions with faithful uncertainty estimation, Nucl. Phys. B 809 (2009) 1 [arXiv:0808.1231] [INSPIRE]. 